\title{
Produção de compósitos madeira-cimento de baixa densidade pelo método vibro-dinâmico de compactação
}

\author{
Production of low density wood-cement \\ composites by vibro-dynamic \\ compaction method
}

\author{
Vinicius Gomes de Castro ${ }^{1}$, Carlos Frederico Alice Parchen ${ }^{1}$,
} Setsuo Iwakiri ${ }^{1}$

\footnotetext{
${ }^{1}$ Departamento de Ciências Agronômicas e Florestais - CCA/UFERSA, Av. Francisco Mota, 572, Bairro Costa e Silva, 59625-900, Mossoró, Rio Grande do Norte, Brasil.

e-mail: vinicius.castro@ufersa.edu.br,parchen@ufpr.br, setsuo@ufpr.br
}

\section{RESUMO}

Esse trabalho teve como objetivo avaliar o potencial de uso da madeira de quatro espécies tropicais da Amazônia na produção de compósitos madeira-cimento pelo método de adensamento por vibro compactação. As partículas de madeira foram produzidas a partir de costaneiras das espécies Murici (Byrsonima crispa), Ingá vermelho (Inga paraensis), Ingá (Inga alba) e Mata-Matá (Eschweilera odora). Todas as espécies foram consideradas aptas para a produção de compósitos de acordo com o teste Fator $\mathrm{C}_{\mathrm{A}}$. Foram testadas as propriedades físicas (densidade, absorção de água e inchamento em espessura) e mecânicas (ligação interna, flexão estática e resistência à compressão) dos compósitos. A densidade da madeira e o tamanho de suas partículas influenciaram a densidade final do compósito e suas propriedades de absorção de água e o módulo de elasticidade. A área superficial da partícula também afetou negativamente a resistência à compressão do material. Tanto o método quanto a madeira apresentaram potencial para fabricação de compósitos com possíveis aplicações como materiais de preenchimento na construção civil

Palavras-chave: Fator $\mathrm{C}_{\mathrm{A}}$, vibro-compactação, correlação de Pearson, madeira tropical.

\section{ABSTRACT}

The aim of this work was evaluate the potential use of timbers from four tropical Amazonian species for production of wood-cement composites by vibro-compaction densification method. Wood particles were made from slab wood of Murici (Byrsonima crispa), Red Inga (Inga paraensis), Inga (Inga alba) e Mata-Mata (Eschweilera odora). All species were considered suitable for composites production according to the $\mathrm{C}_{\mathrm{A}}$ Factor test. Composites physical (density, water absorption and thickness swelling) and mechanical (internal bond, static bending and compressive strength) properties were evaluated. Wood density and particle size influenced the composites final density and their water absorption and modulus of elasticity properties. The particle surface area also affected negatively the material's axial compressive strength. Method and wood showed potential for production of composites with possible application as filling materials in civil construction.

Keywords: $\mathrm{C}_{\mathrm{A}}$ Factor, vibro-compaction, Pearson correlation, tropical wood.

\section{INTRODUÇÃO}

O desenvolvimento das indústrias de compósitos madeira-cimento (CMC) teve início na década de 1930, embora a maior parte das inovações ocorreram nos últimos 40 anos. FRYBORT et al. [1] apresentaram, em sua revisão de literatura sobre esse tipo de material, as principais vantagens dos CMC. Em comparação com madeira sólida e produtos tradicionais de madeira reconstituída, os compósitos apresentam alta estabilidade dimensional, resistência aos agentes biodeterioradores e resistência a fogo. Já comparado com o concreto, a principal vantagem é o fato do CMC ser relativamente mais leve, e isso o torna um produto de boa trabalha- 
bilidade, além de refletir em menores custos de transporte e manuseio.

A principal forma de se controlar a densidade final de um CMC é pela proporção da mistura de seus elementos de formação. Compósitos com maior quantidade de madeira tenderão a apresentar menores densidades nominais. Painéis madeira-cimento comerciais de densidade mínima de $1.100 \mathrm{~kg} \mathrm{~m}^{-3}$ incorporam de 2,75 partes de cimento para uma parte de madeira [2]. Reduzir a relação madeira:cimento para 1:1,55 pode gerar painéis de $600 \mathrm{~kg} \mathrm{~m}^{-3}$, mas torna-se impossível consolidar painéis tão leves quando incorporada uma menor quantidade de cimento em sua composição [3].

É importante conseguir desenvolver alternativas de produções em que a densidade final seja baixa, mas que a proporção de cimento não seja comprometida pois há uma correlação linear entre a razão cimento/madeira e resistência à flexão. Por exemplo, compósitos com relação madeira:cimento de 1:1,75 apresentam menores de módulo de elasticidade do que aqueles feitos com relação de 1:2,5 [4]. Quando uma grande quantidade de madeira é utilizada, a ligação interna também passa a ser comprometida devido a uma menor conectividade entre as partículas de madeira e cimento [5].

PARCHEN et al. [6] apresentaram uma proposta de mudança no método de produção dos compósitos que refletiu na possibilidade de produtos finais mais leves. Ao eliminar a necessidade de prensa, tradicionalmente utilizada para compactação do material, é possível produzir compósitos de densidade próxima a 500 $\mathrm{kg} \mathrm{m}^{-3}$ através da vibro-compactação em uma relação madeira:cimento de 1:1 [6] ou mesmo aumentando o volume de cimento para 1,5 [7]. Uma vez que não há pressão na formação do compósito ao se utilizar a vibro-compactação, a densidade final do produto passa a ser função do volume das partículas de madeira [8]. Em outras palavras, a densidade do produto final é dependente da densidade do material vegetal de origem.

O processo de compactação por vibração consiste essencialmente na eliminação do ar presente na mistura e assentamento das partículas. O problema mais comum que pode ocorrer nos casos de vibração é a compactação não uniforme [9]. A força vibratória é gerada pela combinação de frequiência e amplitude ajustada em vibradores acoplados em caixas formadoras dos compósitos. Frequência é o número de ciclos de vibração por minuto e a amplitude é o tamanho do raio do ciclo de vibratório. Enquanto que a freqüência movimenta partículas menores mais eficientemente, a amplitude age em partículas mais pesadas.

Devido a sua complexa estrutura anatômica, a densidade da madeira pode variar muito de acordo com a espécie botânica. Por exemplo, a madeira sul africana Olea laurifolia é a mais pesada do mundo com densidade de $1.490 \mathrm{~kg} \mathrm{~m}^{-3}$, enquanto que a cubana Aeschynomene hispida é a mais leve com apenas $44 \mathrm{~kg} \mathrm{~m}^{-3}$ [10]. Além da espécie arbórea, a densidade pode variar também dentro de um mesmo indivíduo, uma vez que a madeira é formada por diferentes tecidos com diferentes funções orgânicas na árvore viva. De forma simplificada, a madeira é formada por duas camadas principais: alburno e cerne. O alburno é a parte mais externa ou mais nova do tronco da árvore, onde ocorre transporte de seiva. Está madeira tende a ser mais clara e leve. A parte interna, chamada de cerne, tem a função de sustentação e é formado por tecido fisiologicamente inativo resultante da morte das células do alburno [11].

Outro fator limitante na produção de CMC é a possível inibição que a madeira causa na pega e cura do cimento. Em alguns casos, a madeira pode ser incompatível com o cimento e levar a uma diminuição das propriedades físicas do compósito, podendo criar corpos de prova sem nenhuma integridade [12]. O grau de inibição vai variar de acordo com a composição química da madeira utilizada. Tanto a natureza quanto a quantidade dos componentes químicos da madeira afetam a cura do cimento. Tipicamente os componentes inibitórios podem ser divididos em dois grupos. O primeiro é composto por carboidratos e o segundo de componentes fenólicos [13].

O objetivo deste trabalho foi avaliar o uso da madeira proveniente de resíduos de serrarias da região amazônicas na produção de CMC de baixa densidade pelo método de densificação por vibro-compactação. Para isso, foi avaliado o efeito da madeira de quatro espécies florestais na inibição à pega do cimento e na densificação do compósito. Também foram avaliadas as propriedades físicas e mecânicas dos compósitos de baixa densidade para se determinar o potencial do uso do CMC como material de preenchimento na construção civil.

\section{MATERIAIS E MÉTODOS}

Foram utilizadas madeiras de costaneiras, parte mais externa do tronco considerada resíduo do desdobro em serrarias, de quatro espécies florestais nativas da região amazônica: Murici (Byrsonima crispa Juss.), Ingá vermelho (Inga paraensis Ducke), Ingá (Inga alba (Sw.) Willd) e Mata-Matá (Eschweilera odora (Poepp.) Miers).

As costaneiras foram seccionadas e submersas em água fria por 7 dias antes de serem convertidas em 
partículas. Durante o período de imersão, a água foi trocada diariamente para estimular a extração de substâncias que poderiam atuar como deletérias à pega do cimento. Partículas geradas em um picador de disco foram então secas a um teor de umidade de aproximadamente 3\% e reprocessadas em um moinho de martelo. Após a moagem, as partículas foram classificadas por peneiramento automático.

\subsection{Teste de compatibilidade}

O teste de compatibilidade entre madeira e cimento foi realizado com partículas classificadas em peneiras passantes na malha com $0,84 \mathrm{~mm}$ (20 mesh) de abertura e retidas na de 0,5 mm (35 mesh). Uma quantidade de 90,5 ml de água destilada foi misturada com 200 gramas de cimento Portland tipo CP II-Z e 15 gramas de madeira, seguindo as recomendações de WEATHERWAX e TARKOW [14], que sugerem o uso de $2,7 \mathrm{ml}$ de água por grama de madeira seca e um adicional de $0,25 \mathrm{ml}$ de água por grama de cimento.

Em um recipiente isolado térmica e hermeticamente fechado, foi realizada a leitura das temperaturas através de termopares tipo "J" conectados a um módulo condicionador de sinais analógicos acoplado a uma placa de conversão analógico-digital. As leituras de temperatura foram realizadas a cada minuto durante 24 horas para três repetições de cada tratamento. $\mathrm{O}$ grau de inibição que a madeira causou à pega do cimento foi determinado pelo método Fator- $\mathrm{C}_{\mathrm{A}}$, sugerido por HACHMI e MOSLEMI [15]. Valores de Fator- $\mathrm{C}_{\mathrm{A}}$ acima de 68 indicam uma madeira compatível com o cimento sem necessidade de pré-tratamento, enquanto que valores abaixo de 28 representam espécies incompatíveis. Valores entre 28 e 68 indicam a necessidade de um prétratamento da madeira antes de sua utilização.

\subsection{Produção dos compósitos madeira cimento}

Para a manufatura dos compósitos foram utilizadas partículas passantes em peneira de abertura de 2,36 mm ( 8 mesh) e retidas em abertura de $1,40 \mathrm{~mm}$ (14 mesh). A área superficial das partículas foi calculada pela fórmula sugerida por MOSLEMI [16] com informações das dimensões das partículas obtidas através da média das dimensões de 100 partículas por espécie coletadas aleatoriamente e medidas com o auxilio de um paquímetro digital.

Para a caracterização quantitativa da química das madeiras utilizadas para a produção dos compósitos, foram determinados o teor de extrativos solúveis em água quente [17] e solúveis em Etanol-tolueno 1:2 [18], teor de cinzas [19] e carboidratos de baixo peso molecular solúveis em hidróxido de sódio $(\mathrm{NaOH})$ [20].

O método empregado para manufatura dos compósitos foi o de adensamento por vibro-compactação sugerido por PARCHEN et al. [6]. O cimento utilizado foi o Portland tipo CP II-Z e a mistura de madeira:cimento seguiu a relação 1:2,75 (baseado no peso seco das partículas). Foram adicionados 0,5 ml de água para cada grama de cimento e uma quantidade extra de $0,3 \mathrm{ml}$ para cada grama de madeira seca. $\mathrm{O}$ aditivo químico cloreto de cálcio $\left(\mathrm{CaCl}_{2}\right)$ foi adicionado à mistura, na quantidade de $3 \%$ em relação à massa seca do cimento, como um acelerador da pega do cimento.

As misturas foram depositados em moldes prismáticos feitos de compensado naval com $420 \mathrm{~mm}$ de comprimento, $70 \mathrm{~mm}$ de largura e $15 \mathrm{~mm}$ de espessura, e submetidos à mesa vibratória com uma carga de impacto vertical equivalente a $220 \mathrm{~N}$ à $3.000 \mathrm{RPM}$ por 30 segundos. Com a vibração, ocorreu o adensamento do material e o molde então foi repreenchido com mistura e, novamente, submetido à vibração. Esse processo foi repetido até não haver mais espaço livre no molde mesmo após o período de vibração. Os compósitos foram mantidos dentro do molde por 7 dias. O molde foi então desmontado e os corpos de prova mantidos ao ar livre no período restante de 28 dias necessários para a cura do cimento.

\subsection{Avaliações das propriedades físico-mecânicas}

Os corpos de prova foram cortados com serra diamantada nas dimensões padrões das normas européias e submetidos aos seguintes testes de propriedades físicas e mecânicas: 8 corpos de prova com $50 \mathrm{~mm}$ de largura, $15 \mathrm{~mm}$ de espessura e $350 \mathrm{~mm}$ de comprimento para flexão estática[21], 6 corpos de prova com $50 \mathrm{~mm}$ de largura, $50 \mathrm{~mm}$ de comprimento e $15 \mathrm{~mm}$ de espessura para absorção de água e inchamento em espessura [22] e 8 corpos de prova de $50 \mathrm{~mm}$ de largura, $50 \mathrm{~mm}$ de comprimento e $15 \mathrm{~mm}$ de espessura para ligação interna [23].

Para a resistência à compressão, foram produzidos 7 corpos de prova cilíndricos para cada espécie em moldes de canos PVC de $40 \mathrm{~mm}$ de diâmetro externo e $80 \mathrm{~mm}$ de comprimento. Após 28 dias de cura, os corpos de prova foram ensaiados em uma máquina universal de ensaios com velocidade de carregamento de $0,25 \mathrm{MPa} \mathrm{s}^{-1}$, como indicado pela norma brasileira [24]. 


\subsection{Análise estatística}

A normalidade dos dados foi caracterizada pelos testes Skewness e Kurtosis. Para as sequências de dados que estes testes não observaram uma distribuição normal, foi aplicado o método de Box-Cox de transformação, utilizando o $\lambda$ mais adequado para cada caso. Os dados normalizados foram testados por Análise de Covariância (ANCOVA) e pelo teste de comparação de média Tukey em nível de probabilidade de 5\% de erro considerando a densidade uma covariável.

A medição do grau de intensidade entre duas variáveis aleatórias, que possuem relação linear de causa e efeito entre si, foi feita pela correlação Pearson. Correlações com sinal positivo indicaram uma relação direta, enquanto que o negativo, uma relação inversa.

\section{RESULTADOS}

\subsection{Teste de compatibilidade}

$\mathrm{O}$ teste de compatibilidade Fator- $\mathrm{C}_{\mathrm{A}}$, entre as partículas de madeira após pré-tratamento pelo método da água fria e o cimento Portland tipo II, apresentou valores médios superiores a 68 para todos os tratamentos (Figura 1).

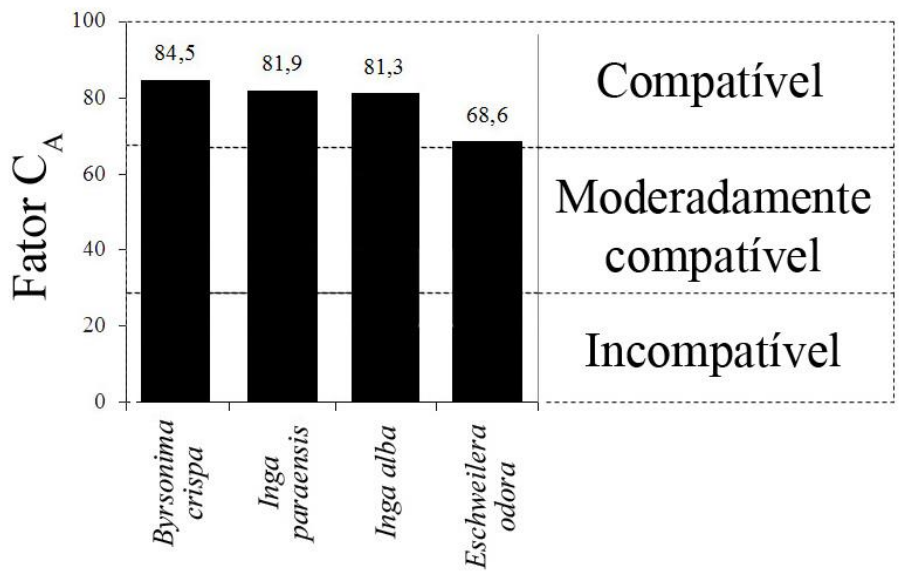

Figura 1: Valores médios do fator $\mathrm{C}_{\mathrm{A}}$ divididos por classes de compatibilidade.

\subsection{Caracterização do material orgânico}

A Figura 2 apresenta as imagens das partículas das quatro espécies utilizadas nas produções dos CMC. 


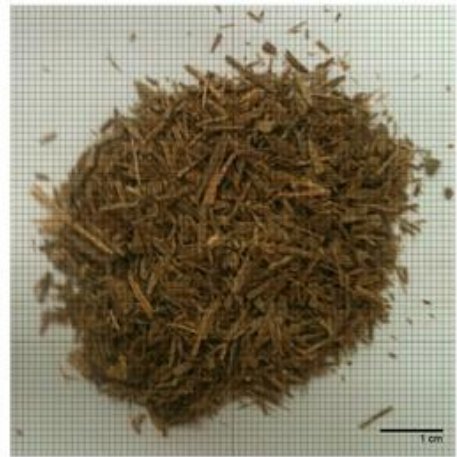

Murici (Byrsonima crispa)

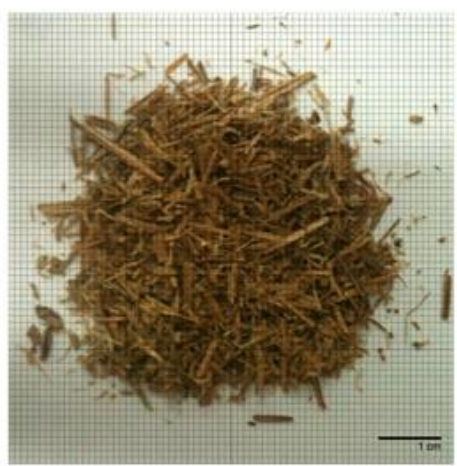

Ingá (Inga alba)

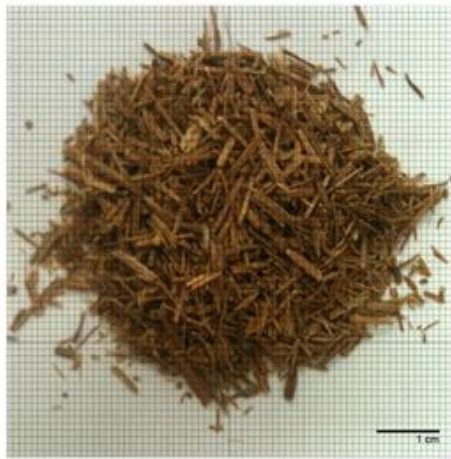

Ingá Vermelho (Inga paraensis)

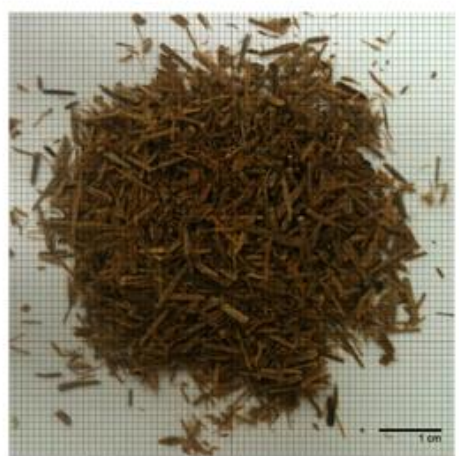

Mata-Matá (Eschweilera odora)

Figura 2: Partículas das quatro espécies de madeira utilizada na produção dos CMC.

As densidades médias das espécies amazônicas variaram entre 570 e $720 \mathrm{~kg} \mathrm{~m}^{-3}$ (Tabela 1), sendo que houve diferença estatisticamente significante entre estes dois valores (das madeiras de Inga alba e Byrsonima crispa, respectivamente). Partículas produzidas com a espécie Inga alba, de menor densidade, foram as únicas que apresentaram área superficial estatisticamente superior as demais.

Tabela 1: Parâmetros físicos das partículas de madeira e a densidade do compósito madeira cimento produzido com estas.

\begin{tabular}{l|l|l|l}
\hline ESPÉCIE & $\begin{array}{l}\text { DENSIDADE DA MADEIRA } \\
\left(\mathbf{k g ~ m}^{-3}\right)\end{array}$ & $\begin{array}{l}\text { ÁREA SUPERFICIAL } \\
\left(\mathbf{c m}^{\mathbf{2}} \mathbf{- 1}\right)\end{array}$ & $\begin{array}{l}\text { DENSIDADE DO COMPÓSITO } \\
\left(\mathbf{k g ~ m}^{-\mathbf{3}}\right)\end{array}$ \\
\hline Byrsonima crispa & $720 \mathrm{~A}$ & $103,4 \mathrm{~B}$ & $1.010 \mathrm{~A}$ \\
\hline Inga paraensis & $630 \mathrm{AB}$ & $108,5 \mathrm{~B}$ & $960 \mathrm{~A}$ \\
\hline Inga alba & $570 \mathrm{~B}$ & $133,9 \mathrm{~A}$ & $900 \mathrm{~B}$ \\
\hline Eschweilera odora & $690 \mathrm{AB}$ & $105,2 \mathrm{~B}$ & $960 \mathrm{~A}$ \\
\hline
\end{tabular}

Nota: Médias seguidas pela mesma letra dentro de uma mesma coluna não diferem estatisticamente pelo teste de Tukey, a $5 \%$ de probabilidade.

A Tabela 2 apresenta os valores médios das propriedades químicas das madeiras utilizadas na produção dos compósitos. A espécie Inga paraensis apresentou maior teor de extrativos hidrossolúvel (água quente) e lipossolúvel (Etanol-tolueno). Também foi a espécie com a maior porcentagem de carboidratos de baixo peso molecular $(1 \% \mathrm{NaOH})$, ou seja, de hemiceluloses e celulose degradada. Em relação a quantidade de materiais inorgânicos presentes na madeira (teor de cinzas), as espécies Byrsonima crispa e Eschweilera odo$r a$ apresentaram valores estatisticamente semelhantes e superiores às demais espécies. 
Tabela 2: Propriedades químicas das madeiras.

\begin{tabular}{l|l|l|l|l}
\hline ESPÉCIE & ÁGUA QUENTE $(\%)$ & ETANOL-TOLUENO $(\boldsymbol{\%})$ & $\mathbf{1 \%} \mathbf{N a O H}(\boldsymbol{\%})$ & TEOR DE CINZAS (\%) \\
\hline Byrsonima crispa & $5,94 \mathrm{C}$ & $3,19 \mathrm{C}$ & $13,32 \mathrm{C}$ & $0,70 \mathrm{~A}$ \\
\hline Inga paraensis & $8,71 \mathrm{~A}$ & $8,36 \mathrm{~A}$ & $17,36 \mathrm{~A}$ & $0,47 \mathrm{~B}$ \\
\hline Inga alba & $7,23 \mathrm{~B}$ & $4,61 \mathrm{~B}$ & $14,71 \mathrm{BC}$ & $0,35 \mathrm{C}$ \\
\hline Eschweilera odora & $6,50 \mathrm{BC}$ & $7,68 \mathrm{~A}$ & $15,18 \mathrm{~B}$ & $0,64 \mathrm{~A}$ \\
\hline
\end{tabular}

Nota: Médias seguidas pela mesma letra dentro de uma mesma coluna não diferem estatisticamente pelo teste de Tukey, a 5\% de probabilidade.

\subsection{Propriedades físico-mecânicas dos CMC}

A Figura 3 apresentada a aparência física dos compósitos produzidos pela mistura cimento e madeiras de espécies amazônicas adensados pelo processo de vibro-compactação.

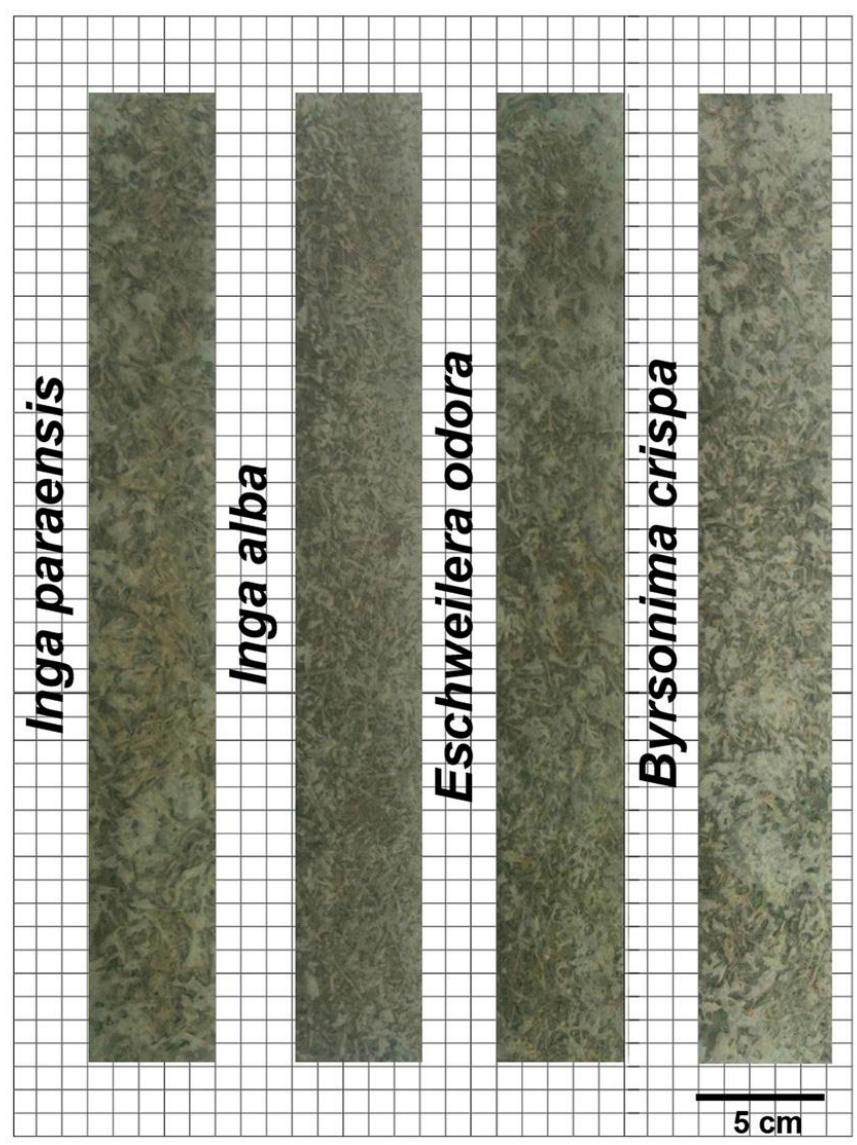

Figura 3: Corpos de prova dos $\mathrm{CMC}$ produzidos com madeiras amazônicas nas dimensões para o teste de flexão estática

A Tabela 3 apresenta os valores médios das propriedades físicas (absorção de água e inchamento em espessura após 2 e 24 horas de imersão) e propriedades mecânicas (ligação interna ou tração perpendicular, módulo de ruptura, módulo de elasticidade e resistência à compressão) .

Tabela 3: Propriedades físicas e mecânicas do CMC produzidos com madeira amazônica por vibro-compactação

PROPRIEDADES FÍSICAS COMPÓSITOS MADEIRA CIMENTO PRODUZIDOS COM:

\begin{tabular}{|l|l|l|l|}
\hline Byrsonima crispa & Inga paraensis & Inga alba & Eschweilera odora
\end{tabular}




\begin{tabular}{|c|c|c|c|c|}
\hline \multirow{2}{*}{$\begin{array}{l}\text { PROPRIEDADES FÍSICAS } \\
\text { Absorção de água } 2 \mathrm{~h}(\%)\end{array}$} & \multicolumn{4}{|c|}{ COMPÓSITOS MADEIRA CIMENTO PRODUZIDOS COM: } \\
\hline & $20,02 \mathrm{~B}$ & $22,46 \mathrm{AB}$ & $27,12 \mathrm{~A}$ & $25,22 \mathrm{AB}$ \\
\hline $\begin{array}{l}\text { Absorção de água } 24 \mathrm{~h} \\
(\%)\end{array}$ & $22,75 \mathrm{~B}$ & $25,25 \mathrm{AB}$ & $29,76 \mathrm{~A}$ & $28,72 \mathrm{AB}$ \\
\hline $\begin{array}{l}\text { Inchamento em espessura } \\
2 \mathrm{~h}(\%)\end{array}$ & $0,21 \mathrm{~A}$ & $0,18 \mathrm{~A}$ & $0,19 \mathrm{~A}$ & $0,16 \mathrm{~A}$ \\
\hline $\begin{array}{l}\text { Inchamento em espessura } \\
24 \mathrm{~h}(\%)\end{array}$ & $0,30 \mathrm{~B}$ & $0,33 \mathrm{~B}$ & $0,54 \mathrm{~A}$ & $0,31 \mathrm{~B}$ \\
\hline \multicolumn{5}{|l|}{$\begin{array}{l}\text { PROPRIEDADES MECÂNI- } \\
\text { CAS }\end{array}$} \\
\hline Ligação interna (MPa) & $0,40 \mathrm{~B}$ & $0,41 \mathrm{~B}$ & $0,58 \mathrm{~A}$ & $0,48 \mathrm{AB}$ \\
\hline $\operatorname{MOR}(\mathrm{MPa})$ & $2,21 \mathrm{~A}$ & $2,42 \mathrm{~A}$ & $2,91 \mathrm{~A}$ & $2,39 \mathrm{~A}$ \\
\hline $\mathrm{MOE}(\mathrm{MPa})$ & $1,403 \mathrm{~A}$ & $1,300 \mathrm{~A}$ & $1,348 \mathrm{~A}$ & $1,292 \mathrm{~A}$ \\
\hline $\begin{array}{l}\text { Resistência à compressão } \\
(\mathrm{MPa})\end{array}$ & $5,98 \mathrm{~A}$ & $7,50 \mathrm{~A}$ & $5,35 \mathrm{~A}$ & $6,71 \mathrm{~A}$ \\
\hline
\end{tabular}

Nota: Médias seguidas pela mesma letra dentro de uma mesma linha não diferem estatisticamente pelo teste de Tukey, a $5 \%$ de probabilidade.

A baixa densidade da madeira de Inga alba e a alta área superficial de suas partículas afetaram a capacidade de absorção de água dos compósitos. Os valores médios de absorção após 2 e 24 horas em compósitos de Inga alba foram estatisticamente superiores. Também houve diferença no inchamento em espessura após 24 horas de imersão.

Em relação às propriedades mecânicas, não houve diferença estatística entre as médias dos compósitos, com exceção da propriedade de ligação interna. Os compósitos produzidos com partículas de Inga alba foram estatisticamente superiores aos produzidos com Byrsonima crispa e Inga paraensis. Contudo, todos os compósitos atenderam ao requisito mínimo de $0,4 \mathrm{MPa}$ para a propriedade de ligação interna sugerido para painéis de madeira-cimento comerciais [2].

A Tabela 4 apresenta a correlação Pearson entre as variáveis referentes as características do material orgânico (fator de compatibilidade entre madeira e cimento; densidade da madeira e área superficial das partículas) e as propriedades físicas e mecânicas avaliadas para os compósitos produzidos por vibrocompactação. Valores seguidos por "*" confirmam uma correlação significativa seja ela direta ou inversa, dependendo do sinal positivo ou negativo, respectivamente.

Tabela 4: Correlação Pearson entre propriedades físico-mecânicas dos compósitos e características da madeira componente.

\begin{tabular}{l|c|c|c}
\hline & FATOR-C $_{\mathbf{A}}$ & DENSIDADE DA MADEIRA & ÁREA SUPERFICIAL \\
\hline Densidade do compósito & 0,2977 & 0,8080 & $-0,08807$ \\
& $(0,4739)$ & $(0,0152)^{*}$ & $(0,0039)^{*}$ \\
\hline Absorção de água & $-0,4185$ & $-0,8519$ & 0,8986 \\
$(2$ horas) & $(0,3021)$ & $(0,0072)^{*}$ & $(0,0024)^{*}$ \\
\hline Absorção de água & $-0,4608$ & $-0,8293$ & 0,8813 \\
(24 horas) & $(0,2505)$ & $(0,0109)^{*}$ & $(0,0038)^{*}$ \\
\hline Inchamento em espessura & $-0,1079$ & 0,6215 & $-0,6385$ \\
$(2$ horas) & $(0,7993)$ & $(0,1000)$ & $(0,0884)$ \\
\hline Inchamento em espessura & $-0,1704$ & $-0,3942$ & 0,4485 \\
$(24$ horas) & $(0,6867)$ & $(0,3339)$ & $(0,2650)$ \\
\hline Ligação interna & $-0,3747$ & $-0,1930$ & 0,2790 \\
& $(0,3604)$ & $(0,6470)$ & $(0,5034)$ \\
\hline
\end{tabular}




\begin{tabular}{l|c|c|c}
\hline & FATOR- $_{\boldsymbol{A}}$ & DENSIDADE DA MADEIRA & ÁREA SUPERFICIAL \\
\hline MOR & 0,3235 & 0,4599 & $-0,4941$ \\
& $(0,4344)$ & $(0,2515)$ & $(0,2133)$ \\
\hline MOE & 0,2585 & 0,7427 & $-0,7377$ \\
& $(0,5365)$ & $(0,0348)^{*}$ & $(0,0367)^{*}$ \\
\hline Resistência à compressão & 0,2531 & 0,6139 & $-0,7295$ \\
& $(0,5453)$ & $(0,1054)$ & $(0,0400)^{*}$ \\
\hline
\end{tabular}

Nota: Valores entre parênteses relativos ao valor-P; * Significativo em nível de probabilidade de $95 \%$.

Embora o Fator- $\mathrm{C}_{\mathrm{A}}$ mensure o efeito da madeira na curva de hidratação do cimento, não pode ser observado nenhuma correlação direta entre o teste de compatibilidade e qualquer das propriedades físicas ou mecânicas dos compósitos produzidos.

A densidade da madeira usada na composição afetou a densidade final do CMC, assim como sua capacidade de absorção de água. Dentre as propriedades mecânicas, só foi observada a correlação entre densidade da madeira e o módulo de elasticidade. E embora a resistência à compressão dos compósitos não tenha sido afetada pela densidade da madeira, houve uma influencia negativamente da área superficial das partículas utilizadas.

\section{DISCUSSÃO}

\subsection{Teste de compatibilidade}

Os valores do Fator- $\mathrm{C}_{\mathrm{A}}$ (Figura 1) indicaram que todas as espécies avaliadas neste trabalho são aptas para a produção de CMC. Espécies de florestas tropicais tendem a ser mais ricas em extrativos do que outras madeiras por isso são muitas vezes erroneamente consideradas incompatíveis com o cimento. Por exemplo, PETTERSEN [25] afirma que o teor de extrativos de madeiras temperadas variam entre 4 e $10 \%$, enquanto que em madeiras tropicais pode chegar a $20 \%$. As espécies analisadas apresentaram valores de extrativos totais (solúveis em água e solvente orgânico) entre 9,13 (Byrsonima crispa) e 17,07\% (Inga paraensis), que são considerados altos.

Os valores médios do Fator- $\mathrm{C}_{\mathrm{A}}$ encontrados para as quatro espécies estudadas foi semelhante aos encontrados para espécies de florestas plantadas. Por exemplo, o Fator- $\mathrm{C}_{\mathrm{A}}$ da espécie folhosa Eucalyptus saligna é 75,2 [8], enquanto que a conífera Pinus taeda é de 85,7 [26]. Estes resultados corroboram com os relatados por CASTRO et al. [27], que não encontraram correlação direta entre a quantidade de extrativos da madeira e os índices de inibição das espécies à pega do cimento. Isso indica que a compatibilidade com o cimento é um processo complexo e espécies de madeira não devem ser descartadas com base apenas em seus teores de extrativos.

A falta de correlação direta pode se resposta da metodologia usada nos testes de compatibilidade. O Fator- $\mathrm{C}_{\mathrm{A}}$ mede a resposta da hidratação do cimento à mistura da madeira em pó, enquanto que na fabricação dos compósitos a madeira é usada em forma de partícula. Segundo KARADE et al. [28], a compatibilidade do pó de madeira não pode ser extrapolada para as condições reais de fabricação de compósitos, embora seja uma informação valiosa pois o efeito da química da madeira seria maior em partículas pequenas e de maior área superficial.

\subsection{Propriedades físico-mecânicas dos CMC}

A densidade das espécies afetou a granulometria das partículas devido ao processo de moagem no moinho de martelo. O material mais leve tende a apresentar menor resistência e gerar partículas menores e consequentemente de maior área superficial. Por sua vez, partículas de Inga Alba, que apresentaram uma área superficial estatisticamente superior as demais partículas, geraram compósitos de menor densidade.

Entretanto, até mesmo os compósitos de menores densidades apresentaram valores médios para as propriedades físicas semelhantes aos apresentados na literatura para CMC produzidos com outras espécies da Amazônia [29], mesmo estes produzidos com prensa hidráulica e com densidades acima de $1.300 \mathrm{~kg} \mathrm{~m}^{-3}$. Os compósitos também apresentaram valores de absorção de água inferiores aos de outros CMC vibrocompactados reportados na literatura [8]. Todos os tratamentos apresentaram valores médios de inchamento 
em espessura aceitáveis, uma vez que recomenda-se para painéis madeira cimento que estes valores mantenham-se abaixo de $1 \%$ após 2 horas de imersão e de 1,5\% após 24 horas de imersão em água [2].

Em relação as propriedades mecânicas, os valores médios para a ligação interna foram superiores a de CMC vibro-compactados que utilizaram partículas de maiores dimensões [6, 7, 30]. PARCHEN et al. [6] encontraram valores inferiores para a propriedade de ligação interna (entre 0,09 e 0,13 MPa) para compósitos produzidos com metodologia e densidade final semelhante ao deste estudo, porém com relação madeira:cimento de 1:1. Por outro lado, CASTRO e IWAKIRI [26] apresentaram valores superiores (1,69 MPa) para a propriedade em compósitos vibro-compactados de baixa densidade mas com maior quantidade de cimento (relação madeira:cimento de 1:4). Por sua vez, para CMC prensados, a ligação interna é influenciada diretamente pelo tamanho da partículas utilizadas e pela quantidade de cimento na mistura [31]. Contudo, para os compósitos vibro-compactados, não foi observada a correlação entre a propriedade de ligação interna e a área superficial das partículas (Tabela 4).

Os valores médios encontrados para as propriedades de MOR e MOE não atenderam os requisitos mínimos para painéis madeira cimento comerciais ( 9 e $3.000 \mathrm{MPa}$, respectivamente) [2]. Porém os resultados foram considerados promissores ao serem comparados com outros compósitos de densidades semelhantes produzidos pelo mesmo método de adensamento. CMC produzidos com partículas de Eucalyptus saligna apresentaram um MOR médio de 1,74 MPa e um MOE de 913 MPa [8]. Já PARCHEN [32] relatou compósitos com partículas de Pinus spp com valores médios de resistência a MOR de 0,29 MPa e MOE de 220 MPa. Os valores médios encontrados para a propriedade de resistência à compressão também foram superiores aos encontrados na literatura para compósitos produzidos com metodologia semelhante, porém com partículas de madeiras maiores $[6,7]$.

\section{CONCLUSÕES}

O uso da madeira de costaneiras de espécies da Amazônia, material considerado resíduo do processo de desdobro em serrarias, é viável para a produção de compósitos madeira-cimento de baixa densidade. O fato de madeiras tropicais apresentarem alto teor de extrativos não deve ser a única variável analisada na escolha da madeira usada na produção, haja visto que não se observou correlações entre o teste de compatibilidade entre madeira e cimento e as propriedades físicas e mecânicas dos compósitos.

O método de vibro-compactação apresentou uma peculiaridade em relação aos CMC produzidos pelo método tradicional de prensagem. Observou-se que a densidade do compósito passa a ser diretamente influenciada pela densidade da madeira. Assim sendo, a escolha de madeira de baixa densidade passam a ser uma opção simples na produção de compósitos madeira-cimento leves, sem que haja a redução da quantidade de cimento na mistura. Desta forma CMC vibro-compactados apresentaram resultados de propriedades mecânicas que, embora sejam inferiores aos exigidos para a fabricação de painéis madeira-cimento tradicionais, os tornam aptos a serem aplicados como possíveis materiais de preenchimento para a construção civil.

\section{AGRADECIMENTOS}

Os autores expressam seus agradecimentos ao Instituto Nacional de Ciência e Tecnologia (INCT)/ Instituto Nacional de Pesquisas da Amazônia (INPA), pela concessão do material objeto desta pesquisa.

\section{BIBLIOGRAFIA}

[1] FRYBORT, S., MAURITZ, R., TEISCHINGER,A., et al., "Cement bonded composites - a mechanical review", Bioresources, v. 3, n. 2, pp. 602-623, 2008.

[2] NCL Industries ltd. Bison panel - Cement bonded particle board. Abids, 2011

[3] OYAGADE, A. O. "Effect of cement/wood ratio on the relationship between cement bonded particleboard density and bending properties", Journal of tropical forest science, v.2, n.2, pp.211-219, 1990.

[4] MARZUKI, A. R., RAHIM, S., HAMIDAH, M., et al., "Effects of wood:cement ratio on mechanical and physical properties of three-layered cement-bonded particleboards from Leucaena leucocephala", Journal of tropical forest science, v. 23, n. 1, pp. 67-72, 2011.

[5] ASHORI, A., TABARSA, T., SEPAHVAND, S. "Cement-bonded composite boards made from poplar strands", Construction and Building Materials, v.26, pp.131-134, 2012.

[6] PARCHEN, C. F. A., IWAKIRI, S., ZELLER, F., et al. "Vibro-dynamic compression processing of lowdensity wood-cement composites", European Journal of Wood and Wood Products, v.74, n.1, pp 75-81, 
2016.

[7] VILLAS-BÔAS, B. T., PARCHEN, C. F. A, IWAKIRI, S., et al. "Avaliação dos efeitos de diferentes dosagens de água/cal na resistência à compressão axial de compósitos de madeira-cimento", Revista Matéria, v. 21, n.1, pp. e11777, 2017.

[8] CASTRO, V. G., BRAZ, R. L., AZAMBUJA, R. R., et al. "Painéis cimento-madeira de Eucalyptus saligna com diferentes aditivos químicos e métodos de formação" Floresta, v.25, pp.349-360, 2015.

[9] NEVILLE, A. M., BROOKS, J.J. Concrete technology, Harlow, Pearson, 2010.

[10] HOADLEY, R. B. Understandig wood, Newtown, Taunton, 2000.

[11] MELO, J. E., CAMARGOS, J. A. A. A madeira e seus usos, Brasília, SFB/LPF/MMA, 2016.

[12] JORGE, F. C., PEREIRA, C., FERREIRA, J. M. F. "Wood-cement composites: a review", Holz als Roh und Werkstoff, v. 62, pp.370-377, 2004.

[13] YASUDA, S., IMA, K., MATSUSHITA, Y. "Manufacture of wood-cement boards VII: cementhardening inhibitory compounds of hannoki (Japanese alder, Alnus japonica Steud.)", Journal of Wood Science, v. 48, pp. 242-244, 2002.

[14] WEATHERWAX, R. C., TARKOW, H. "Effect of wood on setting of Portland cement", Forest Products Journal, v.14, pp.567-570, 1964.

[15] HACHMI, M., MOSLEMI, A. A. "Correlation between wood-cement compatibility and wood extractives”, Forest Products Journal, v.39, pp.55-58, 1989.

[16] MOSLEMI, A. A. "Particle geometry", In: Moslemi, A. A., Particleboard. Vol.1: Material. London, UK, Souther Illinois University Press. pp. 49-67, 1974.

[17] TECHNICAL ASSOCIATION OF THE PULP AND PAPER INDUSTRY (TAPPI) T $207 \mathrm{~cm}-99$. Water solubility of wood and pulp, 1999.

[18] TECHNICAL ASSOCIATION OF THE PULP AND PAPER INDUSTRY (TAPPI) T $207 \mathrm{~cm}$-99. Solvent extractives of wood and pulp, 2007.

[19] TECHNICAL ASSOCIATION OF THE PULP AND PAPER INDUSTRY (TAPPI) T 211 om-02. Ash in wood, pulp, paper and paperbord: combustion at $525^{\circ} \mathrm{C}, 2002$.

[20] TECHNICAL ASSOCIATION OF THE PULP AND PAPER INDUSTRY (TAPPI) T 212 om-02. One percent sodium hydroxide solubility of wood and pulp, 2002.

[21] EUROPEAN COMMITTEE FOR STANDARDIZATION (EN). EN 310: Wood-based panels - Determination of modulus of elasticity in bending and of bending strength. 1993.

[22] EUROPEAN COMMITTEE FOR STANDARDIZATION (EN). EN 317: Aglomerado de partículas de madeira e aglomerado de fibras de madeira - Determinação do inchaço na espessura depois da imersão em água. 1993.

[23] EUROPEAN COMMITTEE FOR STANDARDIZATION (EN). EN 319: Aglomerado de partículas de madeira e aglomerado de fibras de madeira - determinação da força da tensão perpendicular sobre a superfície plana da placa. 1993.

[24] ASSOCIAÇÃO BRASILEIRA DE NORMAS TÉCNICAS (ABNT). NBR 7215: Cimento Portland Determinação da resistência à compressão. 1996.

[25] PETTERSEN, R. C. "The chemical composition of wood", In: ROWELL, R., The chemistry of solid wood. Advances in chemistry series 207. Washington, USA, American Chemical Society. pp.57-126, 1984.

[26] CASTRO, V., IWAKIRI, S. "Influência de diferentes níveis de acetilação nas propriedades físicomecânicas de aglomerados e painéis madeira-cimento", Cerne, v.20, n.4, pp.535-540, 2014.

[27] CASTRO, V. G., AZAMBUJA, R. R., BILA, N. F., et al. "Correlation between chemical composition of tropical hardwoods and wood-cement compatibility", Journal of Wood Chemistry and Technology, v.38, n.1, pp. 28-34, 2017.

[28] KARADE, S. R., IRLE, M., MAHER, K. “Assessment of wood-cement compatibility: a new approach”, Holzforschung, v. 57, pp.672-680, 2003.

[29] MACEDO, A. N., SOUZA, A. A. C., POMPEU NETO, B. B. "Chapas de cimento-madeira com resíduos da indústria madeireira da Região Amazônica”, Ambiente Construído, v.12, n.2, pp.131-150, 2012. 
[30] AZAMBUJA, R. R., CASTRO, V. G., VILLAS-BÔAS, B. T., et al. "Particle size and lime addiction on properties of wood-cement composites produced by the method of densification by vibro compaction", Ciência Rural, v.47, n.6, pp. e20140250, 2017.

[31] SOTANNDE, O. A., OLUWADARE, A. O., OGEDOH, O. et al., "Evaluation of cement-bonded particle board produced from Afzelia africana wood residues", Journal of Engineering Science and Technology, v. 7, n. 6, pp.732-743, 2012.

[32] PARCHEN, C. F. A. Compósito madeira cimento de baixa massa específica produzido com compactação vibro dinâmica., Tese de D.Sc., PPGEF/UFPR, Curitiba, PR, Brasil, 2012.

\section{ORCID}

Vinicius Gomes de Castro

Carlos Frederico Alice Parchen

Setsuo Iwakiri https://orcid.org/0000-0003-0174-0572

https://orcid.org/0000-0001-5890-4772

https://orcid.org/0000-0003-0390-3830 